\title{
Development of performance and error metrics for ultrasound-guided axillary brachial plexus block
}

\author{
Osman MAhmed' \\ Brian D O'Donnell ${ }^{1,2}$ \\ Anthony G Gallagher ${ }^{2}$ \\ George D Shorten ${ }^{1,2}$ \\ 'Department of Anaesthesia and \\ Intensive Care, University College \\ Cork and Cork University Hospital, \\ ${ }^{2}$ The ASSERT Center, University \\ College Cork, Cork, Ireland
}

This article was published in the following Dove Press journal:

Advances in Medical Education and Practice

5 April 2017

Number of times this article has been viewed

Purpose: Change in the landscape of medical education coupled with a paradigm shift toward outcome-based training mandates the trainee to demonstrate specific predefined performance benchmarks in order to progress through training. A valid and reliable assessment tool is a prerequisite for this process. The objective of this study was to characterize ultrasound-guided axillary brachial plexus block to develop performance and error metrics and to verify face and content validity using a modified Delphi method.

Methods: A metric group (MG) was established, which comprised three expert regional anesthesiologists, an experimental psychologist and a trained facilitator. The MG deconstructed ultrasound-guided axillary brachial plexus block to identify and define performance and error metrics. Experts reviewed five video recordings of the procedure performed by anesthesiologists with different levels of expertise to aid task deconstruction. Subsequently, the MG subjected the metrics to "stress testing", a process to ascertain the extent to which the performance and error metrics could be scored objectively, either occurring or not occurring with a high degree of reliability. Ten experienced regional anesthesiologists used a modified Delphi method to reach consensus on the metrics.

Results: Fifty-four performance metrics, organized in six procedural phases and characterizing ultrasound-guided axillary brachial plexus block and 32 error metrics (nine categorized as critical) were identified and defined. Based on the Delphi panel consensus, one performance metric was modified, six deleted and three added.

Conclusion: In this study, we characterized ultrasound-guided axillary brachial plexus block to develop performance and error metrics as a prerequisite for outcome-based training and assessment. Delphi consensus verified face and content validity.

Keywords: task analysis, metrics, validation, training, assessment

\section{Introduction}

Ultrasound-guided regional anesthesia (UGRA) has evolved rapidly to become a fundamental skill for anesthesiologists. ${ }^{1}$ UGRA comprises a group of procedures that facilitate provision of anesthesia and analgesia for different surgical procedures (e.g., upper and lower limbs surgery) either alone or in combination with general anesthesia. It is usually performed by anesthesiologists with advanced level of expertise and requires mastery of a wide range of technical and nontechnical skills. Widespread adoption of UGRA by anesthesiologists has created a requirement to teach and learn UGRA-related skills effectively. Training bodies and groups have endorsed a number of structured training programs to aid skills acquisition during performance of UGRA. ${ }^{2}$ Competence-based procedural skills training has been endorsed and prioritized by
Correspondence: Osman M Ahmed Department of Anaesthesia and Intensive Care, Cork University Hospital, Cork, Ireland

Tel +3532 I492 2I35

Fax +353214546434

Emaildr19osman@icloud.com 
the American and European Societies of Regional Anesthesia. ${ }^{3}$ The current paradigm shift toward competence-based training has led to a demand for objective, valid and reliable assessment tools for procedural skills in general. However, characterization and development of assessment tools for anesthesia-related procedural skills are less advanced compared to their surgical counterparts. ${ }^{4}$ It has been argued that assessment motivates learning and facilitates provision of structured feedback leading to improved procedural skills training. ${ }^{5}$ Assessment of competence could be greatly improved when direct observation with checklists is used. ${ }^{6}$ Disciplines such as surgery have successfully applied a metric-driven proficiency-based progression approach to surgical technical skills training. ${ }^{7}$ This approach (in contrast to generic or Objective Structured Assessment of Technical Skills approach) requires detailed characterization of each specific procedure by unambiguous definition of specific events or "metrics". 8

Procedures such as UGRA may be more effectively taught if mastered initially as component parts, subsequently assimilated into an integrated performance of the complete procedure. ${ }^{9}$ Collaboration with experienced operators who are proficient in performance of the procedure permits identification of 1) what should be done (the essential steps), 2) how it should be done (effective techniques) and 3) what should not be done (errors). This expert-driven procedure characterization must then be subjected to a validation process, for example, face and content validity. ${ }^{10,11}$

The Delphi method is a process that utilizes communication between experts in a field in order to provide a feedback on a given topic. ${ }^{12}$ The Delphi method uses an iterative process for progressing toward a desired result by means of repeated cycles of deliberation. ${ }^{13}$

The objectives of this study were

1. to characterize procedure performance and develop metrics for ultrasound-guided axillary brachial plexus block using qualitative techniques;

2. to verify face and content validity of the metrics using a modified Delphi method.

\section{Methods}

\section{Procedure characterization and metrics generation by metric group}

With approval of the Clinical Research Ethics Committee of the Cork Teaching Hospitals and the expressed consent of all participants, a metric group (MG) composed of three experts (BOD, FL, PM) was established. Experts were defined as anesthesiologists who 1) had undergone formal higher subspecialty training in regional anesthesia, 2) perform ultrasound-guided axillary brachial plexus blocks regularly and 3) currently teach UGRA to trainee anesthesiologists and regional anesthesia fellows. All the three members of the MG are current practicing consultant anesthesiologists and satisfied the aforementioned criteria of an expert. The $\mathrm{MG}$ also included an experimental psychologist (AGG) and a trained facilitator (OMA) who moderated the interactions of the MG to achieve the study objectives.

The investigators defined start and end points for the procedure prior to commencement of the MG meetings. The MG undertook a "task analysis" and deconstruction process, detailed previously by Gallagher et al, ${ }^{8,11}$ to identify units of performance that are integral to skilled performance of ultrasound-guided axillary brachial plexus block. During six face-to-face meetings (each of 120-150 minutes duration) over a 3-month period, performance metrics were identified and then unambiguously defined. The meeting moderator focused on the group interaction to ensure that the definitions derived would enable an independent assessor to subsequently use them with a high degree of reliability. The aims were to 1) make sure that these performance metrics should capture the core elements of procedure performance and 2) create unambiguous operational definitions for each performance metric. Experts included the specific steps and general order (when relevant) in which the procedure should be performed. Procedural phases were specified for groups of related performance metrics. The MG also identified and unambiguously defined potential "error metrics" or actions that deviate from optimal performance. Two types of error metrics are identified within each of the procedure phases. Noncritical errors are behaviors that deviate from optimal performance and might compromise procedure success but do not compromise patient safety. Critical errors are behaviors that deviate from optimal performance and compromise patient safety and might cause actual patient harm. Experts were specifically instructed to characterize a "reference" procedure (i.e., a straightforward procedure) and not an unusual or complex procedure. It was emphasized throughout that the MG should seek to reach consensus, not necessarily agreement between the three experts on the performance and error metrics. Five video recordings of complete live procedures performed by anesthesiologists with different levels of expertise (consultant and trainee anesthesiologists) were reviewed in detail during MG meetings to aid task deconstruction. 


\section{Videography of ultrasound-guided axillary brachial plexus block}

An investigator acquired a video recording of the entire procedure according to specifically predefined start and end points. Written informed consent was obtained from each patient and participating anesthesiologist. Patients were assessed to be ASA I-III scheduled to undergo upper limb orthopedic or plastic surgery procedure, and for whom an axillary brachial plexus block was planned, by the responsible consultant anesthesiologist as part of their perioperative management.

Two cameras were used to record the procedure: firstperson perspective imaging was obtained using a headmounted camera (GoPro ${ }^{\mathrm{TM}}$ Hero GP 1049 [Patented USA] GoPro Inc., San Mateo, CA, USA) placed on operator's head; third-person perspective imaging was obtained by the investigator using a handheld camera (Sony HX5V HD; Sony Corporation of America, New York, NY, USA). Concurrent ultrasound video images were recorded using the DVR feature of the Ultrasonix tablet (Sonix Design Centre BK Ultrasound, Richmond, BC, Canada). All video recordings were edited using iMovie (Apple iMovie, version 10.0.5; Apple Computer Inc., Cupertino, CA, USA) to present the procedure, from first- or third-person perspective as appropriate, together with the ultrasound output on one screen. This was achieved using "picture-in-picture" mode (Figure 1). The ultrasound feed was displayed on the right side of the screen, taking care not to obscure the view of the operator's hands. Both the procedure and ultrasound video recordings were time synchronized. The identities of patient and participant were masked. These video recordings were reviewed by the MG to facilitate their effort to ensure that the defined metrics were both observable and valid.

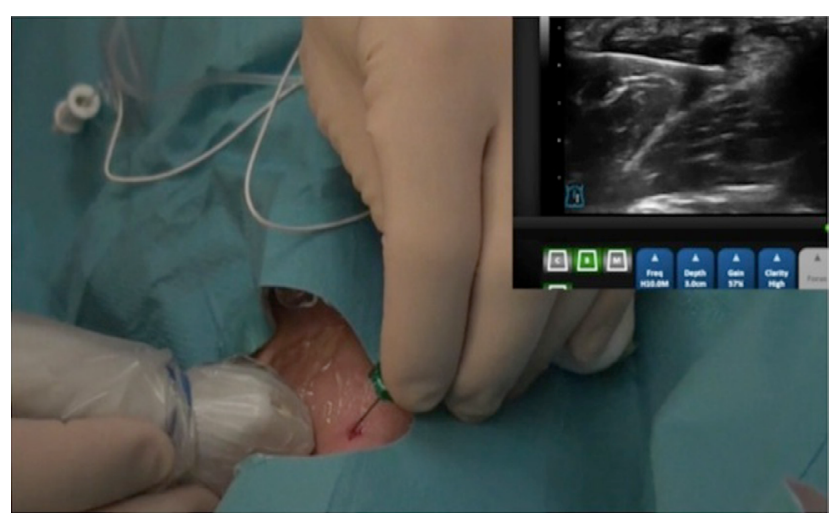

Figure I A view from a video recording presented to the MG to aid procedure characterization. Views are from the operative procedure and ultrasound output. Abbreviation: MG, metric group.

\section{Performance and error metrics reliability assessment "stress testing" by MG}

Following the "task analysis" phase, the MG examined the performance and error metrics for reliability testing "Stress Testing", ${ }^{8,11}$ Each of the three members, independently, reviewed and scored two video recordings of the procedure that were acquired during the metrics development stage, in a blinded fashion. After viewing and scoring the recordings, scoring patterns from all the three experts were compared and discussed in a joint and final (seventh) MG meeting.

\section{Examination of face and content validity using a modified Delphi method}

Once the MG members were satisfied that all procedure phases were appropriately characterized, the performance and error metrics were subjected to face and content validity testing using a modified Delphi panel method.

Ten consultant anesthesiologists each of whom was a member of Irish Society of Regional Anaesthesia and who are also experts in regional anesthesia (as per the aforementioned criteria) participated in this process. Members of the MG, who had originally developed the metrics, did not participate in the Delphi panel.

A brief overview of the study and meeting objectives were presented by one of the investigators. Background information regarding task analysis, procedure characterization and the specific objectives of the current Delphi panel were presented. Each phase of the procedure and the associated performance and error metrics were presented. As part of this briefing, it was emphasized that the designated "reference procedure" was not intended to reflect the exact technique employed by an individual expert practitioner, but to show that the performance metrics presented were intended to accurately capture the essential and key components of the procedure. The specific questions asked were: 1) Do these metrics represent a reasonable characterization of the procedure? 2) Do any of the metrics represent an incorrect approach? Members of the Delphi panel were also asked if a performance or error metric definition should be modified or if a metric should be added or deleted. After each of the procedure phases was presented, attendees were given time to review individual metrics in detail. After each metric definition was reviewed, panel members voted on whether or not the metric was acceptable as written. An affirmative vote by a panel member indicated that the metric definition presented was accurate and acceptable as written. If consensus could not be achieved, then modifications as suggested 
by the panel were employed and members re-voted until consensus was achieved.

\section{Results}

The performance metrics developed (categorized in six procedural phases) are summarized in Table 1. Error metrics (noncritical and critical) are summarized in Table 2.

During the final (seventh) MG meeting, the three members subjected the performance and error metrics to "stress testing". The outcome was a final modification of two metrics relating to maintaining asepsis, which were condensed to appear after section $E$ (metrics 42, 43; Table 1) rather than after each of the preceding sections.

The Delphi panel meeting was held on May 21, 2015, during the Irish Congress of Anesthesia (ICA) in Dublin. The meeting lasted for 180 minutes. Three phases (B, F and $\mathrm{E}$ ) attained consensus with a single round of voting and no modifications were made. Three phases (A, C and D) required modifications and subsequently achieved consensus after a second round of voting. The amendments that were derived from the Delphi panel meeting are as follows:

Phase A: Four members voted against performance metric 6 (Table 1) so that it was modified to "sedation to be administered as clinically indicated" (not on patient request) and subsequently attained consensus in the second round of voting.

Phase C: Six members voted to delete two performance metrics; one about wearing a facemask and the other about using povidone-iodine (Betadine) as an antiseptic; the consensus was to delete these. Members also voted to delete metric 21 (Table 1) as not needed for single shot block, but it was subsequently retained following the second round of deliberation; members discussed and subsequently voted on adding performance metric 22 (Table 1).

Phase D: All members agreed that performance metrics related to operator verbalizing their interpretation of ultrasonography scan (four performance metrics) would introduce a distraction. Following further discussion, members reached a consensus to evaluate that phase of the procedure based on two specific "outcome" performance metrics (metrics 28, 29; Table 1).

\section{Discussion}

In this study, we characterized skilled performance of ultrasound-guided axillary brachial plexus block. Face and content validity of the outcome of the task characterization (metrics) had been examined and confirmed using a modified
Table I Six phases of the procedure characterization and abbreviated summary of the definitions of the 54 performance metrics developed by the MG and validated by the Delphi panel

I. Place patient on a suitable trolley or bed

2. Establish conversation with the patient

3. Perform safe surgical checklist

4. Establish an intravenous cannula

5. Establish monitoring

6. Give sedative medication

7. Administer supplemental oxygen

B. Procedure ergonomics

8. Adjust equipment within workplace

9. Remove clothing from arm

10. Expose patients arm

II. Place arm in block position

C. Aseptic preparation (patient, operator, equipment)

12. Ensure operator arms are bare

13. Decontaminates hands

14. Wear sterile gloves

15. Prepare block tray

16. Prepare a high-frequency ultrasound probe

17. Prepare local anesthetics solution

18. Confirm name and expiry date of local anesthetics

19. Flush block needle

20. Decontaminate skin of the axilla

21. Establish sterile field by using sterile drapes

22. Fix sterile drapes

D. Ultrasonography

23. Check sidedness of the ultrasound probe

24. Place probe on the axilla

25. Hold probe in a tripod grip

26. Confirm identity of axillary artery

27. Immobilize the probe

28. Acquire an optimum ultrasound image

29. Verbalize the main components of the optimum ultrasound image

E. Needle guidance and injectate placement

30. Inform the patient of needle insertion

31. Advance block needle adjacent to ultrasound probe

32. Keep needle visible when advancing

33. Reestablish needle visibility during foreword motion

34. Withdraw needle toward skin to reestablish visibility

35. Maintain communication with patient

36. Aspirate before injection of local anesthetics

37. Maintain patient veins during injection

38. Encourage patient to report pain on injection

39. Use test dose of local anesthetics

40. Place full block dose

4I. Place sufficient amount of local anesthetics

42. Maintain sterility of procedure field

43. Correct breaches of sterility if required

F. Block clinical assessment

44. Remove cast or splint from the blocked arm

45. Establish presence or absence of pain

46. Assess motor power along distribution of median nerve

47. Assess motor power along distribution of ulnar nerve

48. Assess motor power along distribution of radial nerve proximal fibers

49. Assess motor power along distribution of radial nerve distal fibers 50. Assess motor power along distribution of musculocutaneous nerve

5I. Assess sensory function along distribution of median nerve

52. Assess sensory function along distribution of ulnar nerve

53. Assess sensory function along distribution of radial nerve

54. Assess sensory function along distribution of musculocutaneous nerve

Abbreviation: MG, metric group. 
Table 2 Abbreviated summary of the definitions of the 32 (noncritical and critical) errors developed during procedure characterization

I. Procedure room (block room) is not prepared for performing the block

2. Not running conversation with the patient

3. No monitoring attached (critical)

4. No intravenous cannula sited (critical)

5. Not administering oxygen (critical)

6. Adopt an awkward posture during block performance

7. Patient dignity not maintained

8. Causing patient pain during positioning

9. Personal apparel not removed

10. Antiseptic solution not dried before needle insertion

II. Failure to maintain asepsis (critical)

12. Incorrect placement of local skin infiltration

13. Advance needle other than infiltrated skin area

14. Advance needle out of plane

15. Veins are compressed during injection

16. Failure to visualize needle

17. Advance needle without tip being visible

18. Continuously move needle to reestablish needle visibility

19. Multiple needle redirections without established visibility

20. Require multiple skin puncture during procedure

21 . Failure to ask patient about pain and paresthesia

22. Failure to aspirate before injection

23. Failure to use test dose

24. Place less than sufficient amount of local anesthetic

25. Continue to inject despite incorrect site of injection

26. Failure to place local anesthetic around each nerve

27. Advance needle despite patient reporting pain (critical)

28. Continue to inject despite evidence of intraneural injection (critical)

29. Place block dose without visual evidence of injectate (critical)

30. Continue to inject despite aspirating blood (critical)

3I. Place needle into a nerve (critical)

32. Not performing block assessment

Delphi method. In addition to the specific new performance and error metrics developed, the study describes in detail the application of this methodology to an image-guided procedure with patient needle interaction for the first time.

Procedural skills play an important role in anesthetic practice. Evidence-based training and assessment of procedural skills are necessary as training moves from an apprenticeship-based model to one of competency-based training. ${ }^{14}$ Our results are consistent with previous studies. Angelo et al ${ }^{15}$ adopted a similar methodology to characterize task and develop performance and error metrics for arthroscopic shoulder surgery. Subsequently, they successfully incorporated the metrics into a training curriculum in simulated learning environments. ${ }^{16}$ For ultrasound-guided nerve blocks, checklists and global rating scales (GRS) have been developed and examined for validity. Both Cheung et $\mathrm{al}^{17}$ and Chuan et a ${ }^{18}$ developed a "one-size-fits-all" assessment tool for UGRA to include checklist and GRS and validated the tool by using a modified Delphi method. GRS as used in these two studies permits a degree of subjectivity, which may influence the assessment outcome. This subjectivity in turn hampers interrater reliability, an essential component of a valid assessment tool. ${ }^{19}$ The methodology used in this study requires detailed characterization of the procedure by generating unambiguously defined performance and error metrics and their subsequent examination for face and content validity. This approach has been quantitatively shown to have greater assessment reliability when compared to Likert scale assessments used with GRS. ${ }^{19}$ One of the strengths of this study is that each performance and error metric represents an observable behavior that is precisely defined within the context of ultrasound-guided axillary brachial plexus block. We believe that this will minimize ambiguity and enhance objectivity in the assessment process. Hence, we expect a high interrater reliability when used by trained observers. The ultimate goal will be to objectively determine whether specific behaviors (metric or error) have occurred or not.

This study provided one prerequisite element of an evidence-based approach to training ultrasound-guided axillary brachial plexus block, namely, the development of an assessment tool. More work will be necessary to integrate this into an overall proficiency-based training program and to prospectively examine the resulting performance effect versus the best alternative currently available. The proficiency-based progression approach mandates that the trainee be able to demonstrate the ability to meet specific predefined benchmarks to progress in training. These benchmarks must have specific, clear, objective and fair standards of performance. The metrics that we described in this study will be essential in defining these standards. The importance of this study is that it provides data and a framework for the development of such a standard (although this was beyond the scope of this study).

It is also important for the task analysis to identify behaviors that deviate from optimal performance, i.e., "error metrics". It has been proven that error-focused checklists are superior to conventional checklists in terms of evaluating procedural incompetence..$^{20}$ Any training program based on the performance and error metrics generated in this study will need to be evaluated (versus the best available alternative) in terms of any influence in incidence of defined error metrics in both simulated and clinical settings.

Various forms of checklists have been applied to assessment of procedural and nontechnical skills. What differentiates the approach described in this study is the rigor and methodological detail applied to generating, defining and 
stress testing of each performance and error metric. We believe that the resulting unambiguous definitions effectively "capture" the critical elements of performance and underpin the efficacy of subsequently developed training programs. In the past (when applied to surgical procedures), the training efficacy of such programs has been invariable and substantial.

The study has potential weaknesses. The three experts in the MG are very experienced in the performance of ultrasound-guided axillary brachial plexus block. We acknowledge that three (experts) is a small number of sources from which to elicit details of a reference procedure, which is performed in many different setting around the world. The fact that all the three experts work in a single center raises the possibility that their views represent or contain some institutional bias. These limitations are mitigated by the use of the Delphi process, proven to be an excellent and reliable method to test expert view for consistency with that of a wider practicing community. Another important limitation of this study relates to the definition of metrics as observable behaviors when a substantial part of the procedure entails cognitive elements that are not "observable". Much interpretation of the initial ultrasound scan is based on cognitive function, which is not directly captured as observable behavior. We judged that prompting the operator to verbalize their interpretation or reasoning during the ultrasound image acquisition would introduce a distraction and an unwarranted demand on cognitive resources ${ }^{21}$ and may cause an operator to deviate from his/her intended or normal practice. Based on the Delphi panel discussion, it was decided to evaluate that phase of the procedure based on two specific "outcome" metrics $(28$, 29; Table 1) to minimize distraction caused by prompting.

We also acknowledge the need to examine the set of performance and error metrics described here for construct validity and reliability. This has been investigated recently using a prospective observational design. ${ }^{22}$ Future studies are required to evaluate the effectiveness of new training programs based on these metrics.

\section{Conclusion}

In summary, we applied a methodology (successful in surgical technical skills training) to characterize performance of ultrasound-guided axillary brachial plexus block. The performance and error metrics presented here have face and content validity and may be used to objectively assess trainee skills acquisition and facilitate feedback during training. Integration of these into proficiency-based progression training program will require definition of performance benchmarks and prospective examination of the novel training program versus the best alternative.

\section{Acknowledgment}

The authors thank Dr Frank Loughnane and Dr Padraig Mahon for their contributions during the metrics development. They were members of the MG and participated in the identification and definition of metrics and errors.

\section{Author contributions}

Conception and design of study: OMA, BDO, AGG, and GDS; data acquisition, analysis and interpretation: OMA, BDO, AGG, and GDS; and drafting, revising and approval of the manuscript: OMA, BDO, AGG, and GDS. All the authors agreed to be accountable to all aspects of publication process.

\section{Disclosure}

The authors report no conflicts of interest in this work.

\section{References}

1. Marhofer P, Greher M, Kapral S. Ultrasound guidance in regional anesthesia. Br J Anaesth. 2005;94(1):7-17.

2. Regional Anesthesiology and Acute Pain Medicine Fellowship Directors Group. Guidelines for fellowship training in regional anesthesiology and acute pain medicine: second edition, 2010. Reg Anesth Pain Med. 2011;36(3):282-288

3. Sites BD, Chan VW, Neal JM, et al. The American Society of Regional Anesthesia and Pain Medicine and the European Society of Regional Anaesthesia and Pain Therapy joint committee recommendations for education and training in ultrasound-guided regional anesthesia. Reg Anesth Pain Med. 2010;35(2 Suppl):S74-S80.

4. Bould MD, Crabtree NA, Naik VN. Assessment of procedural skills in anaesthesia. Br J Anaesth. 2009;103(4):472-483.

5. Epstein RM. Assessment in medical education. N Engl J Med. 2007; 356(4):387-396.

6. Regehr G, MacRae H, Reznick RK, Szalay D. Comparing the psychometric properties of checklists and global rating scales for assessing performance on an OSCE-format examination. Acad Med. 1998;73(9):993-997.

7. Gallagher AG, Ritter EM, Champion H, et al. Virtual reality simulation for the operating room: proficiency-based training as a paradigm shift in surgical skills training. Ann Surg. 2005;241(2):364-372.

8. Gallagher AG. Metric-based simulation training to proficiency in medical education:- what it is and how to do it. Ulster Med J. 2012; 81(3):107-113.

9. Brydges R, Carnahan H, Backstein D, Dubrowski A. Application of motor learning principles to complex surgical tasks: searching for the optimal practice schedule. J Mot Behav. 2007;39(1):40-48.

10. Gallagher AG, Ritter EM, Satava RM. Fundamental principles of validation, and reliability: rigorous science for the assessment of surgical education and training. Surg Endosc. 2003;17(10):1525-1529.

11. Gallagher AG, O'Sullivan GC. Fundamentals of Surgical Simulation: Principle and Practice. London: Springer-Verlag; 2011.

12. Morgan PJ, Lam-McCulloch J, Herold-Mcllroy J, Tarshis J. Simulation performance checklist generation using the Delphi technique. Can $J$ Anaesth. 2007;54(12):992-997.

13. Clayton MJ. Delphi: a technique to harness expert opinion for critical decision-making tasks in education. Educ Psychol. 1997;17(4):373-386. 
14. RCOA. CCT in anaesthetics IV: competency based higher and advanced level (2007). The Royal College of Anaesthetists 2016. Available at: http://www.rcoa.ac.uk/document-store/cct-anaesthetics-iv-competency-based-higher-and-advanced-level-2007. Accessed February 6, 2016.

15. Angelo RL, Ryu RK, Pedowitz RA, Gallagher AG. Metric development for an arthroscopic Bankart procedure: assessment of face and content validity. Arthroscopy. 2015;31(8):1430-1440.

16. Angelo RL, Pedowitz RA, Ryu RK, Gallagher AG. The Bankart performance metrics combined with a shoulder model simulator create a precise and accurate training tool for measuring surgeon skill. Arthroscopy. 2015;31(9):1639-1654.

17. Cheung JJ, Chen EW, Darani R, McCartney CJ, Dubrowski A, Awad IT. The creation of an objective assessment tool for ultrasound-guided regional anesthesia using the Delphi method. Reg Anesth Pain Med. 2012;37(3):329-333.
18. Chuan A, Graham PL, Wong DM, et al. Design and validation of the Regional Anaesthesia Procedural Skills Assessment Tool. Anaesthesia. 2015;70(12):1401-1411.

19. Gallagher AG, O'Sullivan GC, Leonard G, Bunting BP, McGlade KJ. Objective structured assessment of technical skills and checklist scales reliability compared for high stakes assessments. ANZ J Surg. 2014;84(7-8):568-573.

20. Ma IW, Pugh D, Mema B, Brindle ME, Cooke L, Stromer JN. Use of an error-focused checklist to identify incompetence in lumbar puncture performances. Med Educ. 2015;49(10):1004-1015.

21. Gallagher AG, Satava RM, O’Sullivan GC.Attentional capacity: an essential aspect of surgeon performance. Ann Surg. 2015;261(3):e60-e61.

22. Ahmed OM, O'Donnell BD, Gallagher AG, Breslin DS, Nix CM, Shorten GD. Construct validity of a novel assessment tool for ultrasound-guided axillary brachial plexus block. Anaesthesia. 2016; 71(11):1324-1331.
Advances in Medical Education and Practice

\section{Publish your work in this journal}

Advances in Medical Education and Practice is an international, peerreviewed, open access journal that aims to present and publish research on Medical Education covering medical, dental, nursing and allied health care professional education. The journal covers undergraduate education, postgraduate training and continuing medical education

\section{Dovepress}

including emerging trends and innovative models linking education, research, and health care services. The manuscript management system is completely online and includes a very quick and fair peer-review system. Visit http://www.dovepress.com/testimonials.php to read real quotes from published authors.

Submit your manuscript here: http://www.dovepress.com/advances-in-medical-education-and-practice-journal 